\title{
Autonomous Upper and Lower Elevator Security Robot Based on Machine Vision
}

\author{
Haoyang $\mathrm{Xu}^{\text {a) }}$, Zheming Yang ${ }^{\text {b) }}$ and Qiuhong Jia ${ }^{\mathrm{c})}$ \\ North China University of Science and Technology, Tangshan 063000, China. \\ a) superxhy@qq.com \\ b) yangzheminga@qq.com \\ c)2834281917@qq.com
}

\begin{abstract}
The security robot realizes the detection of the elevator contour and key by the machine vision algorithm and realizes the control of the elevator door with the manipulator, so as to realize the independent elevator. On the same floor, it achieves the best path and then cruises through path planning and operates the elevator buttons through the manipulator to go up and down the stairs. In case of a critical situation such as a fire, the robot will sound and emit light to alert. It can also achieve positioning through mobile phone APP, start or stop patrols, one-click homing and other functions.
\end{abstract}

Keywords: Machine vision; Elevator Robot; Security; Artificial intelligence.

\section{INTRODUCTION}

With the rapid development of the information age, security robots have become a hot research object in the information industry. [1] Robots have also become more intelligent. However, there is still no public security robot that can be promoted to autonomously go up and down the elevator. Robotics integrates many disciplines such as machinery, information, and intelligent control. It not only has high added value of its own technology, but also has a wide range of product applications, and it has become an important technology radiation platform. It is of great significance to enhancing military defense capabilities, improving the handling of emergencies, [2] stimulating overall economic development, and improving people's living standards.

\section{OVERALL SYSTEM DESIGN}

The robot is composed of an elevator identification and key operating system, a [3] power mobile system, a positioning and route planning system in the building, and a real-time monitoring system for smoke, fire and obstacle avoidance, as shown in Fig. 1.

[4] The elevator recognition and key operating system uses the matching graphics function in OpenCV to find and locate elevator keys. In order to ensure the good linearity of the car, the power transmission system uses two motors to drive the left and right wheels. And on its back end is equipped with a universal wheel, so that the robot can get better mobility and flexibility. We set up some RFID markers on the corridors, bends, and stairs to achieve robot positioning. When the robot is moving, it detects in real time whether an RFID tag has passed through the RFID reader. If the robot passes an RFID tag, we can read the unique number of the RFID tag to determine the robot's current position, as shown in Fig. 2. 


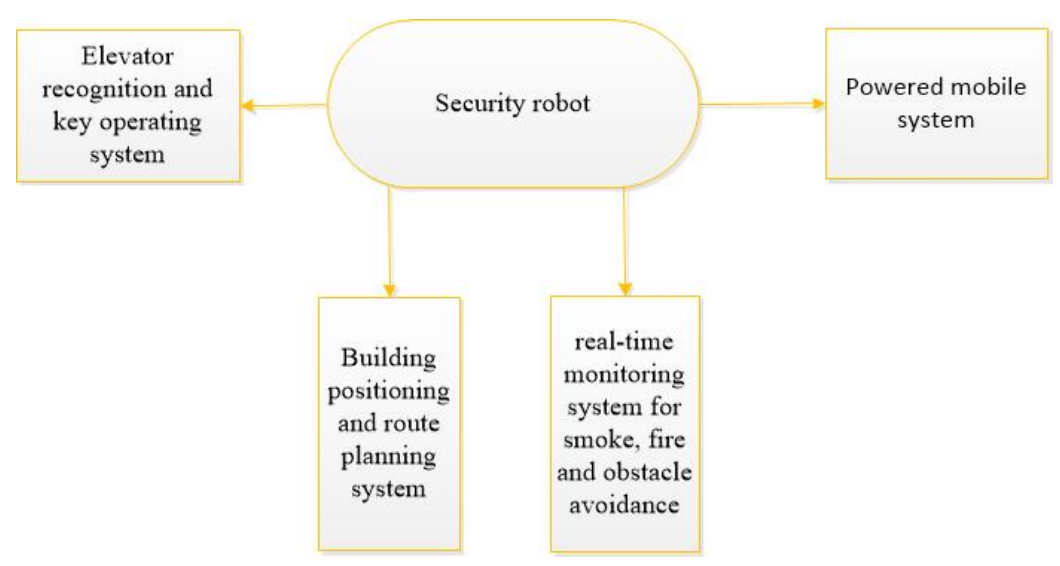

FIGURE 1. Overall System Design

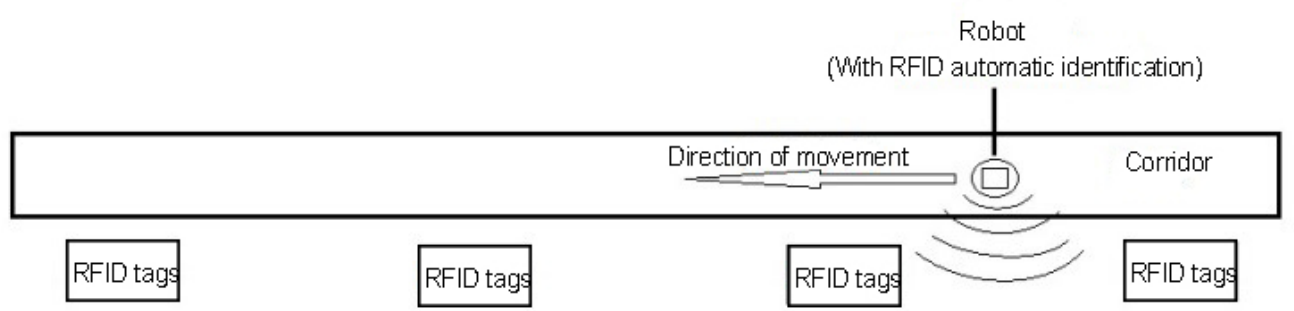

FIGURE 2. RFID Indoor Positioning Technology

The [5] route planning system adopts a round-trip route planning method. When the robot is placed indoors, it can avoid obstacles in real time through sensors such as laser radar and ranging module. Not only that, we also connected robots to the Internet through the ESP8266 to realize the interconnection of mobile phones APP and robots.

\section{DESIGN OF ELEVATOR SECURITY ROBOT}

\section{Elevator Recognition Algorithm}

The shape of the elevator and the appearance of the elevator buttons are unchanged once they are determined, which is very advantageous for us to find and locate elevator buttons based on the images collected by the camera. So we chose to use template [6] matching technology.

(1) Loads an input image returned by the camera and a [7] template image block.

(2) The template matching technique requires that the template image block and input image have the same proportion. However, the size of the input image will change as the camera of the robot moves away from the elevator. Therefore, we need to change the image size and then perform template matching. Due to the large size of the input image, scaling it up consumes system resources, so the template image block should be scaled. We use a [8] step-by-step scaled multiple from small to large $(0.1,0.2,0.3 \ldots)$ to run a template match after each zoom.

(3) We use the function match Template to implement any of the six matching methods. We can choose any kind of test method until getting better recognition results in the test phase.

(4) We normalize the matched results to get a match rate between 0 and 1 . When the matching rate reaches 0.8 or more, elevator key recognition is successful. If not, it means there is no elevator button in the camera image.

(5) If the elevator key recognition is successful, the best matching area is located, and the coordinates of a template image block positioned on the image are output.

(6) Use a [9] rectangular box to mark the best match area. 
We use the camera to take a picture of the elevator and use an algorithm to process it. The final recognition is successful. The results are shown in Fig. 3.

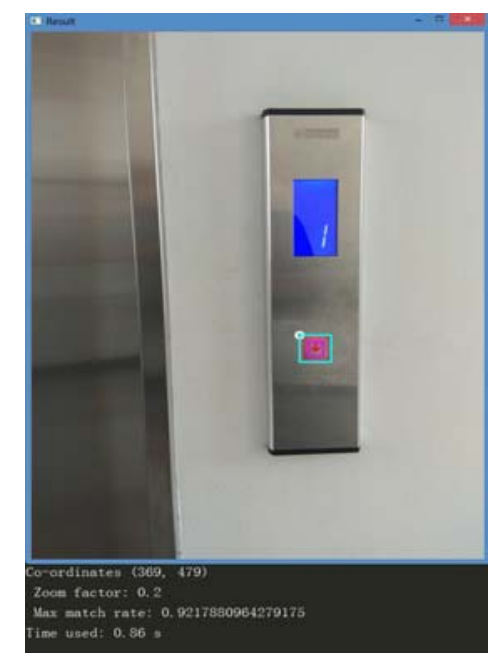

FIGURE 3. Results Schematic

\section{Arm Operation}

\section{Mechanical Structure of the Robot Arm}

Due to the large number of moving parts of the robot, its movement state often changes, and shock and vibration are inevitable. Therefore, it is especially important to increase the robot motion [10] stability and the robot dynamics. Under the premise of meeting the strength and stiffness, in order to reduce the quality of the moving parts, we used a three-degrees-of-freedom robotic arm to realize the operation of elevator keys, as shown in Fig. 4. This can ensure that the robot can perform physical operations in a certain space around, and also ensure the reliability and lightness of the structure.

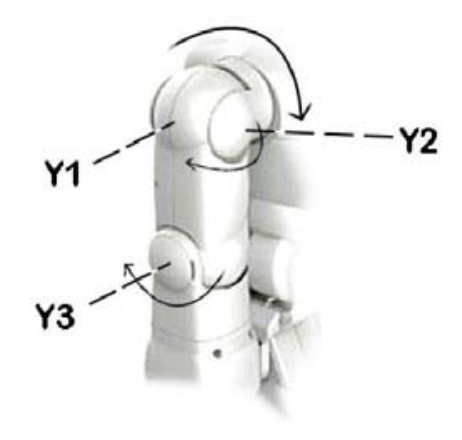

FIGURE 4. Arm Structure Diagram

\section{Manipulator Motion Control}

In order to improve the accuracy of the robotic operation, we use the [11] negative feedback motion algorithm (PID). We enter the difference between the coordinates of the end of the manipulator in the camera and the coordinates of the target elevator key. With the PID algorithm, we can get the coordinates that the end of the manipulator should reach. With the constant adjustment of the coordinates, the end of the manipulator finally presses the target button. After that, in combination with the target position identified by the image, the movement 
of the end of the arm is controlled to operate the elevator key. Therefore, the robot can autonomously control the elevator keys and go up and down the stairs.

\section{Robot Autonomously Operates the Elevator}

When the robot's program determines that the floor is to be replaced, the robot constantly changes its position to the elevator via the RFID positioning system. Next, the camera of the robot monitors the screen in real time and determines the coordinate position of elevator keys by visual algorithm. Then the robot [12] controls the robot arm to operate the elevator button and uses the laser radar and distance measuring module in front to detect whether the elevator door is open. After the elevator door is opened, the robot enters the elevator and rotates $180^{\circ}$ in place so that the robot faces the elevator door. And it identifies each key in the elevator and monitors the coordinate position of the corresponding key. The robot's patrol procedure determines the next floor that the robot will go to and presses the corresponding key on the floor with the mechanical arm. When the elevator reaches the [13] destination floor, and the robot's distance sensor detects that the elevator door is open, the robot can walk out of the elevator.

\section{CONCLUSION}

The security robot is not only smart and efficient, but also adaptable. It also comes with an elevator identification system that can autonomously go up and down the stairs. Besides, its ACS anti-collision system can protect robots and people from damage. Its mode is flexible and it also has high security. The robot is flexible, convenient, highly integrated, and has many functions. Therefore, it can be used in personal homes, warehouses, schools and other places. It not only provides convenience for people's lives, but also saves labor costs. Based on the above, it has a broad market prospects, in line with today's people's requirements for life intelligence.

\section{REFERENCES}

1. Control of a direct drive robot using fuzzy spiking neural networks with variable structure systems-based learning algorithm [J]. Yesim Oniz,Okyay Kaynak. Neurocomputing . 2014.

2. Topology Control for Connectivity Maintenance in Cooperative Mobile Robot Networks [J]. Sidney R.D. Carvalho, Filipe L. de Barros Correia, Ubirajara F. Moreno. IFAC Papers on Line. 2015 (19).

3. Knightscope's K5 Robot Stops Crime Before It Happens [J]. Wiltz, Chris. Design News . 2015 (3).

4. Robot Technology. Graham, Ian. 2012.

5. Video camera with anti-shake system. Katoh H, Ichinoi Y. US 7525572 B2. 2009.

6. Airplane cbb/security camera system interface. Poblete D D. US 20030117494 A1. 2003.

7. Improving Robustness in Multi-Robot Networks. Cinara G, Cristian S, Carlos H C R, et al. IFAC-Papers on Line. 2015.

8. "Model-Predictive Motion Planning,". T.M. Howard, M. Pivtoraiko, R.A. Knepper, A. Kelly. IEEE Robotics Automation Magazine. 2014.

9. Designing winning robots by careful design of their development process [J] Eli Kolberg, Yoram Reich, Ilya Levin. Research in Engineering Design. 2014 (2).

10. An auction behavior-based robotic architecture for service robotics [J]. Bradford A. Towle, Monica Nicolescu. Intelligent Service Robotics. 2014 (3).

11. Smart talking robot Xiaotu: participatory library service based on artificial intelligence [J]. Fei Yao, Chengyu Zhang, Wu Chen. Library Hi Tech . 2015 (2).

12. Consistent Steering System using SCTP for Bluetooth Scatternet Sensor Network [J]. R. Dhaya, V. Sadasivam, R. Kanthavel. Journal of The Institution of Engineers (India): Series B. 2013 (4).

13. A modular design of Bayesian networks using expert knowledge: Context-aware home service robot [J]. HanSaem Park, Sung-Bae Cho. Expert Systems With Applications . 2011 (3). 